\title{
Dark Energy density in Split SUSY models inspired by degenerate vacua
}

\section{Colin Froggatt}

Glasgow University

E-mail: C.froggattephysics.gla.ac.uk

Roman Nevzorov*i

University of Hawaii

E-mail: nevzorov@phys.hawaii.edu

\section{Holger Bech Nielsen}

The Niels Bohr Institute

E-mail: hbech@nbi.dk

It is well known that global symmetries protect local supersymmetry and a zero value for the cosmological constant in no-scale supergravity. The breakdown of these symmetries, which ensure the vanishing of the vacuum energy density, results in a set of degenerate vacua with broken and unbroken supersymmetry leading to the natural realisation of the multiple point principle (MPP). Assuming the degeneracy of vacua with broken and unbroken SUSY in the hidden sector we estimate the value of the cosmological constant. We argue that the observed value of the dark energy density can be reproduced in the split-SUSY scenario if the SUSY breaking scale is of the order of $10^{10} \mathrm{GeV}$.

35th International Conference of High Energy Physics

July 22-28, 2010

Paris, France

\footnotetext{
* Speaker.

${ }^{\dagger}$ On leave of absence from the Theory Department, ITEP, Moscow, Russia.
} 


\section{No-scale supergravity and the multiple point principle}

Recent observations indicate that $70 \%-73 \%$ of the energy density of the Universe exists in the form of dark energy. This tiny vacuum energy density (the cosmological constant) $\Lambda \sim 10^{-123} M_{P l}^{4} \sim$ $10^{-55} M_{Z}^{4}$ is responsible for the accelerated expansion of the Universe. In the standard model (SM) the cosmological constant is expected to be many orders of magnitude larger than the observed vacuum energy density. An exact global supersymmetry (SUSY) ensures zero value for the vacuum energy density. However the breakdown of SUSY induces a huge and positive contribution to the cosmological constant of order $M_{S}^{4}$, where the SUSY breaking scale $M_{S} \gg 100 \mathrm{GeV}$.

In general the vacuum energy density in $(N=1)$ supergravity (SUGRA) models is huge and negative $\Lambda \sim-m_{3 / 2}^{2} M_{P l}^{2}$, where $m_{3 / 2}$ is the gravitino mass. The situation changes dramatically in no-scale supergravity where the invariance of the Lagrangian under imaginary translations and dilatations results in the vanishing of the vacuum energy density. Unfortunately these global symmetries also protect supersymmetry which has to be broken in any phenomenologically acceptable theory. The breakdown of dilatation invariance does not necessarily result in a non-zero vacuum energy density [1]-[2]. This happens if the dilatation invariance is broken in the superpotential of the hidden sector only. The hidden sector of the simplest SUGRA model of this type involves two singlet superfields, $T$ and $z$. The invariance under the global symmetry transformations constrains the Kähler potential and superpotential of the hidden sector, which can be written in the following form [1]:

$$
\hat{K}=-3 \ln \left[T+\bar{T}-|z|^{2}\right], \quad \hat{W}(z)=\kappa\left(z^{3}+\mu_{0} z^{2}+\sum_{n=4}^{\infty} c_{n} z^{n}\right) .
$$

Here we use standard supergravity mass units: $\frac{M_{P l}}{\sqrt{8 \pi}}=1$. The bilinear mass term for the superfield $z$ and the higher order terms $c_{n} z^{n}$ in the superpotential $\hat{W}(z)$ spoil the dilatation invariance. However the SUGRA scalar potential of the hidden sector remains positive definite in the considered model

$$
V(T, z)=\frac{1}{3\left(T+\bar{T}-|z|^{2}\right)^{2}}\left|\frac{\partial \hat{W}(z)}{\partial z}\right|^{2},
$$

so that the vacuum energy density vanishes near its global minima. In the simplest case when $c_{n}=0$, the scalar potential (1.2) has two extremum points at $z=0$ and $z=-\frac{2 \mu_{0}}{3}$. In the first vacuum, where $z=-\frac{2 \mu_{0}}{3}$, local supersymmetry is broken and the gravitino gains a non-zero mass. In the second minimum, the vacuum expectation value of the superfield $z$ and the gravitino mass vanish.

Thus the considered breakdown of dilatation invariance leads to a natural realisation of the multiple point principle (MPP). The MPP postulates the existence of many phases allowed by a given theory having the same energy density [B] $-[$ [ $[$. In SUGRA models of the above type there is a vacuum in which the low-energy limit of the considered theory is described by a pure supersymmetric model in flat Minkowski space. According to the MPP this vacuum and the physical one in which we live must be degenerate. Such a second vacuum is only realised if the SUGRA scalar potential has a minimum where $m_{3 / 2}=0$ which normally requires an extra fine-tuning [5]. In the SUGRA model considered above the MPP conditions are fulfilled automatically without any extra fine-tuning at the tree-level. We now assume the existence of a phenomenologically viable model of this type having the physical vacuum degenerate with a second supersymmetric flat vacuum. 


\section{The value of the dark energy density}

Since the vacuum energy density of supersymmetric states in flat Minkowski space is just zero and all vacua in the MPP inspired SUGRA models are degenerate, the cosmological constant problem in the physical vacuum is thereby solved to first approximation by our assumption. However non-perturbative effects in the observable sector can give rise to the breakdown of SUSY in the supersymmetric state (phase) at low energies. Then the MPP assumption implies that the physical phase in which local supersymmetry is broken in the hidden sector has the same energy density as the phase where supersymmetry breakdown takes place non-perturbatively in the observable sector.

In order to simplify our analysis we restrict our consideration by taking the simplest canonical form for the gauge kinetic functions $f_{a}(T, z) \simeq$ const. Due to the mild dependence of $f_{a}(T, z)$ on $T$ and $z$ the gauginos of mass $M_{a}$ are typically substantially lighter than the scalar particles of mass $m_{\alpha}$ in the considered SUSY models, i.e. $M_{a} \ll m_{\alpha} \sim m_{3 / 2}$. Such a hierarchical structure of the particle spectrum naturally appears in Split Supersymmetry models.

If supersymmetry breaking takes place non-perturbatively in the second vacuum, it is caused by the strong interactions. When $f_{a}(T, z) \simeq$ const, the gauge couplings at high energies are almost identical in both vacua and their running down to the SUSY breaking scale $M_{S} \sim m_{3 / 2} \sim m_{\alpha}$ are also the same. Then using the matching condition $\alpha_{3}^{(2)}\left(M_{S}\right)=\alpha_{3}^{(1)}\left(M_{S}\right)$, one finds the scale $\Lambda_{S Q C D}$, where the supersymmetric QCD interactions become strong in the second vacuum:

$$
\Lambda_{S Q C D}=M_{S} \exp \left[\frac{2 \pi}{b_{3} \alpha_{3}^{(2)}\left(M_{S}\right)}\right], \quad \frac{1}{\alpha_{3}^{(2)}\left(M_{S}\right)}=\frac{1}{\alpha_{3}^{(1)}\left(M_{Z}\right)}-\frac{\tilde{b}_{3}}{4 \pi} \ln \frac{M_{g}^{2}}{M_{Z}^{2}}-\frac{b_{3}^{\prime}}{4 \pi} \ln \frac{M_{S}^{2}}{M_{g}^{2}} .
$$

In Eq.(2.1) $\alpha_{3}^{(1)}$ and $\alpha_{3}^{(2)}$ are the values of the strong gauge couplings in the physical and second vacua respectively, $M_{g}$ is the gluino mass, while $\tilde{b}_{3}=-7, b_{3}=-3$ and $b_{3}^{\prime}=-5$ are the oneloop beta functions for the strong gauge coupling in the SM, MSSM and Split SUSY scenario respectively. At the scale $\Lambda_{S Q C D}$ the t-quark Yukawa coupling in the MSSM is of the same order of magnitude as the strong gauge coupling. The large Yukawa coupling of the top quark may result in the formation of a quark condensate that breaks supersymmetry, inducing a non-zero positive value for the cosmological constant $\Lambda \simeq \Lambda_{S Q C D}^{4}$.

In Fig. 1 the dependence of $\Lambda_{S Q C D}$ on the SUSY breaking scale $M_{S}$ is examined. We set $M_{g}=500 \mathrm{GeV}$. From Fig. 1 one can see that the value of $\Lambda_{S Q C D}$ is much lower than the QCD scale in the Standard Model and diminishes with increasing $M_{S}$. The measured value of the cosmological constant is reproduced when $M_{S} \sim 10^{10} \mathrm{GeV}$. With increasing gluino mass the value of the SUSY breaking scale that results in an appropriate value of the cosmological constant decreases. The results of our numerical analysis indicate that for $\alpha_{3}\left(M_{Z}\right)=0.116-0.121$ and $M_{g}=500-2500 \mathrm{GeV}$ the value of $M_{S}$ varies from $2 \cdot 10^{9} \mathrm{GeV}$ up to $3 \cdot 10^{10} \mathrm{GeV}$.

The obtained prediction for the supersymmetry breaking scale can be tested. A striking feature of the Split SUSY model is the extremely long lifetime of the gluino. In the considered case the gluino decays through a virtual squark to $q \bar{q}+\chi_{1}^{0}$. The large mass of the squarks then implies a long lifetime for the gluino. This lifetime is given by

$$
\tau \sim 8\left(\frac{M_{S}}{10^{9} \mathrm{GeV}}\right)^{4}\left(\frac{1 \mathrm{TeV}}{M_{g}}\right)^{5} s .
$$




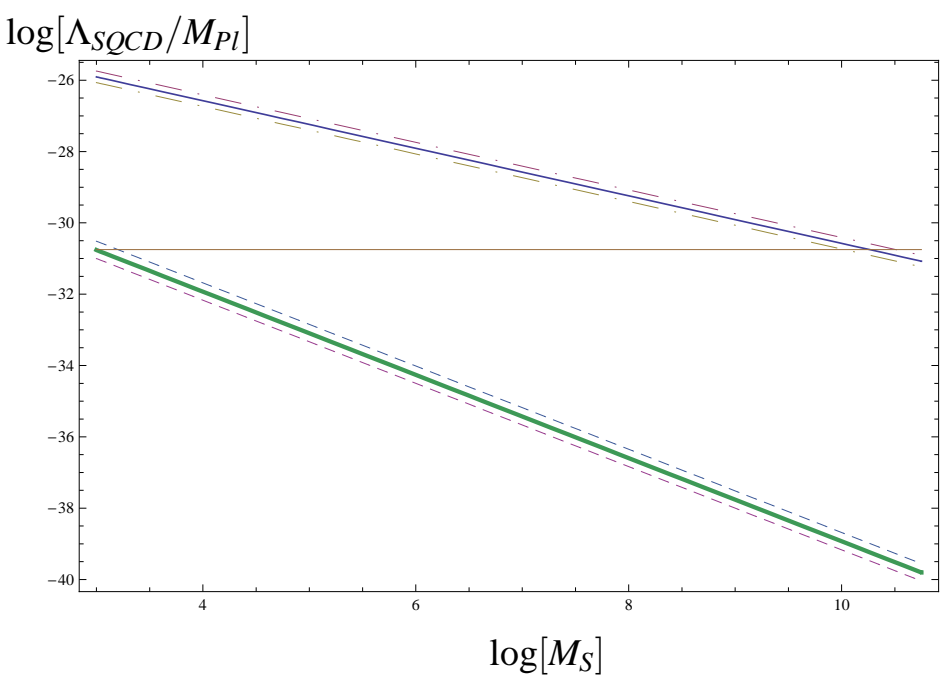

Figure 1: The value of $\log \left[\Lambda_{S Q C D} / M_{P l}\right]$ versus $\log M_{S}$ for $M_{q}=M_{g}=500 \mathrm{GeV}$. The thin and thick solid lines correspond to the Split SUSY scenarios with the pure MSSM particle content and the MSSM particle content supplemented by an additional pair of $5+\overline{5}$ multiplets respectively. The dashed and dash-dotted lines represent the uncertainty in $\alpha_{3}\left(M_{Z}\right)$. The thin and thick solid lines are obtained for $\alpha_{3}\left(M_{Z}\right)=0.1184$, the upper (lower) dashed and dash-dotted lines correspond to $\alpha_{3}\left(M_{Z}\right)=0.116\left(\alpha_{3}\left(M_{Z}\right)=0.121\right)$. The horizontal line represents the observed value of $\Lambda^{1 / 4}$. The SUSY breaking scale $M_{S}$ is measured in GeV.

If, as is predicted, the SUSY breaking scale lies in the interval from $2 \cdot 10^{9} \mathrm{GeV}\left(M_{g}=2500 \mathrm{GeV}\right)$ to $3 \cdot 10^{10} \mathrm{GeV}\left(M_{g}=500 \mathrm{GeV}\right)$ the corresponding gluino lifetime lies in the interval from $1 \mathrm{sec}$. to $2 \cdot 10^{8} \mathrm{sec}$. (1000years). Thus the measurement of the gluino lifetime will allow an estimate to be made of the value of $M_{S}$ in the Split SUSY model.

The observed cosmological constant can also be reproduced for a much lower SUSY breaking scale, if the MSSM particle content is supplemented by an additional pair of $5+\overline{5}$ supermultiplets. In the physical vacuum new bosonic states associated with the extra $5+\overline{5}$ multiplets gain masses around $M_{S}$ while their fermionic partners can have masses of the order of the gluino mass. So we set the masses of the new vectorlike quarks $M_{q}$ equal to $M_{g}$. Then from Fig. 1 it is easy to see that the observed value of the cosmological constant can be reproduced even for $M_{S} \simeq 1 \mathrm{TeV}$.

\section{References}

[1] C. Froggatt, R. Nevzorov and H. B. Nielsen, On the smallness of the cosmological constant in SUGRA models, Nucl. Phys. B $\mathbf{7 4 3}$ (2006) 133 [hep-ph/0511259].

[2] C. Froggatt, L. Laperashvili, R. Nevzorov and H. B. Nielsen, No-scale supergravity and the multiple point principle, [hep-ph/0411273].

[3] D. L. Bennett and H. B. Nielsen, Predictions for nonAbelian fine structure constants from multicriticality, Int. J. Mod. Phys. A 9 (1994) 5155 [hep-ph/9311321].

[4] D. L. Bennett, C. D. Froggatt and H. B. Nielsen, Nonlocality as an explanation for fine tuning and field replication in nature, [hep-ph/9504294].

[5] C. Froggatt, L. Laperashvili, R. Nevzorov and H. B. Nielsen, Cosmological constant in SUGRA models and the multiple point principle, Phys. Atom. Nucl. 67 (2004) 582 [hep-ph/0310127]. 